\title{
IN VIVO PHENOTYPIC CHARACTERISATION OF NUCLEOSIDE LABEL-RETAINING CELLS IN MOUSE PERIOSTEUM
}

\author{
H. M. Cherry, A. J. Roelofs, T. B. Kurth ${ }^{\S}$ and C. De Bari*
}

Arthritis Research UK Tissue Engineering Centre, UK, and Regenerative Medicine Group, Musculoskeletal Research Programme, Institute of Medical Sciences, University of Aberdeen, Scotland, UK

\begin{abstract}
Periosteum is known to contain cells that, after isolation and culture-expansion, display properties of mesenchymal stromal/stem cells (MSCs). However, the equivalent cells have not been identified in situ mainly due to the lack of specific markers. Postnatally, stem cells are slow-cycling, long-term nucleoside-label-retaining cells. This study aimed to identify and characterise label-retaining cells in mouse periosteum in vivo. Mice received iodo-deoxyuridine (IdU) via the drinking water for 30 days, followed by a 40 -day washout period. IdU+ cells were identified by immunostaining in conjunction with MSC and lineage markers. IdU-labelled cells were detected throughout the periosteum with no apparent focal concentration, and were negative for the endothelial marker von Willebrand factor and the pan-haematopoietic marker CD45. Subsets of IdU+ cells were positive for the mesenchymal/stromal markers vimentin and cadherin-11. IdU+ cells expressed stem cell antigen-1, CD44, CD73, CD105, platelet-derived growth factor receptor- $\alpha$ and $p 75$, thereby displaying an MSC-like phonotype. Co-localisation was not detectable between IdU and the pericyte markers CD146, alpha smooth muscle actin or NG2, nor did IdU co-localise with $\beta$-galactosidase in a transgenic mouse expressing this reporter gene in pericytes and smooth muscle cells. Subsets of IdU+ cells expressed the osteoblast-lineage markers Runx 2 and osteocalcin. The IdU + cells expressing osteocalcin were lining the bone and were negative for the MSC marker $\mathrm{p} 75$. In conclusion, mouse periosteum contains nucleoside-label-retaining cells with a phenotype compatible with MSCs that are distinct from pericytes and osteoblasts. Future studies characterising the MSC niche in vivo could reveal novel therapeutic targets for promoting bone regeneration/repair.
\end{abstract}

Keywords: Mesenchymal stem/stromal cell; osteoprogenitor; periosteum; label-retaining; osteoblast; pericyte.

\author{
$\S$ Deceased \\ *Address for correspondence: \\ Cosimo De Bari \\ Institute of Medical Sciences \\ Foresterhill, Aberdeen AB25 2ZD, UK
}

Telephone Number: +44 1224437477

Email: c.debari@abdn.ac.uk.

\section{Introduction}

Periosteum, the thin membranous tissue situated at the boundary between bone and the surrounding soft tissues, has long been known to be osteogenic. As early as 1867, the French surgeon Ollier reported that free periosteal grafts are able to produce bone (Ollier, 1867). It is now well-established that periosteum, in particular the cambium layer facing the bone, is a rich source of stem/ progenitor cells which play important roles in bone repair. Removal of the periosteum significantly diminishes the capacity for repair both in animal models (Ozaki et al., 2000) and clinically (Gerstenfeld et al., 2003).

Fibroblast-like cells can be isolated from periosteum that display properties of mesenchymal stromal/stem cells (MSCs), including clonogenicity, phenotypic marker expression, and ability to differentiate into osteoblasts, chondrocytes, and adipocytes (Nakahara et al., 1990; Nakahara et al., 1991; De Bari et al., 2001; De Bari et al., 2006). However, the equivalent cells have not been identified in vivo due to lack of specific markers to identify these cells in their native tissue. Therefore, many questions remain regarding the topography, phenotype, function and regulation of MSCs in periosteum and the niches they reside in. A full understanding of the biology of stem/progenitor cells in periosteum is critical for the development of improved protocols and discovery of novel pharmacological targets to prevent and treat fractures.

Adult stem cells are conventionally considered as being slow-cycling cells. Hence, long-term labelretaining assays and associated marker analyses have been successfully employed to identify stem cells in their own tissue environments, such as in the bulge of the hair follicle (Cotsarelis et al., 1990; Tumbar et al., 2004). We recently demonstrated that functional MSCs in mouse synovium are label-retaining cells (Kurth et al., 2011). Studies in the 1960s using tritiated thymidine showed that periosteum also contains label-retaining cells (Tonna et al., 1960; Tonna and Cronkite et al., 1962; Tonna and Cronkite, 1968). However, the phenotype of these cells was not fully determined due to lack of available phenotypic markers at that time. Long-term label-retaining cells are not limited to slow-cycling stem cells, but also include cells that, following labelling, undergo differentiation into a postmitotic mature phenotype. Hence, there is a requirement for marker analyses to identify label-retaining cells with an MSC phenotype in vivo. In this study, we performed a classical long-term nucleoside-analogue-retaining assay and determined the phenotype and topography of the label-retaining cells in periosteum by immunostaining for conventional MSC and lineage markers. 


\section{Materials and Methods}

Animals and nucleoside analogue administration Animal experiments were approved by the UK Home Office and conducted in accordance with the Animals (Scientific Procedures) Act 1986 and the Home Office Code of Practice. Twenty-one-day-old male C57BL/6 mice and transgenic mice expressing the LacZ gene in pericytes and smooth muscle cells (Tidhar et al., 2001) received the artificial nucleoside iodo-deoxy-uridine (IdU; SigmaAldrich, Gillingham, UK) with the drinking water for 30 days at a concentration of $1 \mathrm{mg} / \mathrm{mL}$. IdU administration was then stopped for the next 40 days (washout period), after which mice were killed.

\section{Histological sample processing}

Femora were dissected and fixed in $2 \%$ paraformaldehyde and $0.05 \%$ glutaraldehyde in PBS for $1 \mathrm{~h}$ at room temperature. After decalcification for 2 weeks in $4 \%$ ethylenediaminetetraacetic acid (EDTA) in phosphatebuffered saline (PBS), samples were dehydrated and paraffin-embedded. Five- $\mu \mathrm{m}$ thick longitudinal sections through the distal femur were obtained and placed on Superfrost ${ }^{+}$slides (Menzel, Braunschweig, Germany).

\section{Immunostaining}

Immunohistochemical detection of IdU was performed as described previously (Kurth et al., 2011), with the modification that the secondary antibody was visualised using 3,3-diaminobenzidine peroxidase substrate, and sections were counterstained with Haematoxylin QS and eosin. Double and triple immunofluorescence stainings to detect IdU in conjunction with MSC and lineage markers were performed using antibodies and optimised protocols as reported previously (Kurth et al., 2011). In addition, rabbit anti-NG2 (Millipore-Chemicon, Temecula, CA, USA, AB5320) was used at 1:100 dilution following antigen retrieval by boiling in an EDTA-based buffer solution ( $\mathrm{pH} \mathrm{9)}$ for $30 \mathrm{~min}$, goat anti-mouse osteocalcin (AbD serotec 7060-1815) was used at 1:100 dilution following antigen retrieval by pepsin $(0.5 \mathrm{mg} / \mathrm{mL})$ in $0.2 \mathrm{~N} \mathrm{HCl}$ for $30 \mathrm{~min}$, and goat anti-mouse runx 2 (Abcam, Cambridge, UK; ab102712) was used at 1:50 dilution following antigen retrieval by boiling in an EDTA-based buffer solution ( $\mathrm{pH}$ 9) for $30 \mathrm{~min}$. Isotype negative controls were included in all immunostainings to exclude nonspecific antibody binding. Sections were mounted using Mowiol containing 4',6-diamidino-2-phenylindole (DAPI) for nuclear counterstaining. Images were acquired from the perichondrial groove of Ranvier, the metaphysis and diaphysis of the periosteum using a Zeiss (Jena, Germany) Imager fluorescence microscope equipped with a Zeiss ApoTome, or a Zeiss LSM700 laser-scanning confocal microscope. Representative images are shown.

\section{Quantification of labelled cells}

A minimum of 3 sections per mouse from 3 mice underwent immunofluorescence staining for IdU and the MSC marker p75, chosen because it is not expressed by osteoblasts. Nuclei were counterstained with DAPI. From each section, a minimum of 3 images of the perichondrial groove of
Ranvier, the metaphyseal and the diaphyseal periosteum were obtained. At least 500 total DAPI-stained cells per mouse were counted. Values were expressed as average \pm standard deviation (SD) $(n=3)$ of the percentage of IdU positive cells, p75 positive cells or IdU/p75 double-positive cells within the total number of DAPI-counterstained cells, and the percentage of IdU/p75 double-positive cells within the IdU positive cells or the p75 positive cells.

\section{Results}

\section{Nucleoside-label-retaining cells are present throughout mouse periosteum}

To detect nucleoside-label-retaining cells in mouse periosteum, three-week-old male $\mathrm{C} 57 \mathrm{Bl} / 6$ mice were given the nucleoside analogue IdU for 30 days followed by a 40-day washout period. We opted to perform the labelling assay in young, rapidly growing, mice to increase specificity of the assay in the distinction between longterm label-retaining slow-cycling (stem) cells and the cells that instead proliferate extensively to support animal growth. IdU was then detected by immunohistochemistry in longitudinal sections through the distal femur (Fig. 1A). Three anatomical areas of periosteum were distinguished, namely the periosteum lining the perichondrial groove of Ranvier, the metaphyseal periosteum, and the diaphyseal periosteum. IdU+ cells were detected scattered in all three areas of the periosteum with no apparent focal concentration (Fig. 1B-D).

\section{Nucleoside-label-retaining cells in periosteum have an MSC phenotype}

The phenotype of the label-retaining cells in periosteum was determined by co-immunostaining for IdU and MSC or lineage markers. IdU+ cells were non-endothelial and non-haematopoietic, as they were negative for the endothelial marker von Willebrand factor (vWF; Fig. 2A) and the pan-haematopoietic marker CD45 (Fig. 2B), respectively. Subsets of IdU+ cells were positive for cadherin-11 (Fig. 2C) and vimentin (Fig. 2D), markers of mesenchymal/stromal cells (Dou et al., 2012; Guler et al., 2012; Kaur et al., 2012). Next, we determined whether IdU+ cells in periosteum expressed any of the conventional MSC markers, focussing first on markers reported to be expressed on either mouse or human uncultured MSCs. Stem-cell-associated antigen 1 (Sca-1) and platelet-derived growth factor receptor alpha (PDGFR $\alpha$, also known as CD140a) have been described as positive markers to identify freshly isolated MSCs from mouse bone marrow (Peister et al., 2004; Morikawa et al., 2009). Co-staining for IdU and Sca-1 revealed subsets of IdU+ cells expressing this MSC marker (Fig. 2E). Similarly, a subset of IdU+ cells co-stained for PDGFR $\alpha$ (Fig. 2F). Immunofluorescence staining for IdU together with p75, also known as lowaffinity nerve growth factor receptor (LNGFR) or CD271, and described as a marker of freshly isolated human bone marrow MSCs (Jones et al., 2002; Tormin et al., 2011), also revealed double-positive cells in mouse periosteum (Fig. 2G). Cells double-positive for IdU and the MSC markers Sca-1, PDGFR $\alpha$, or p75 were frequently found 
A

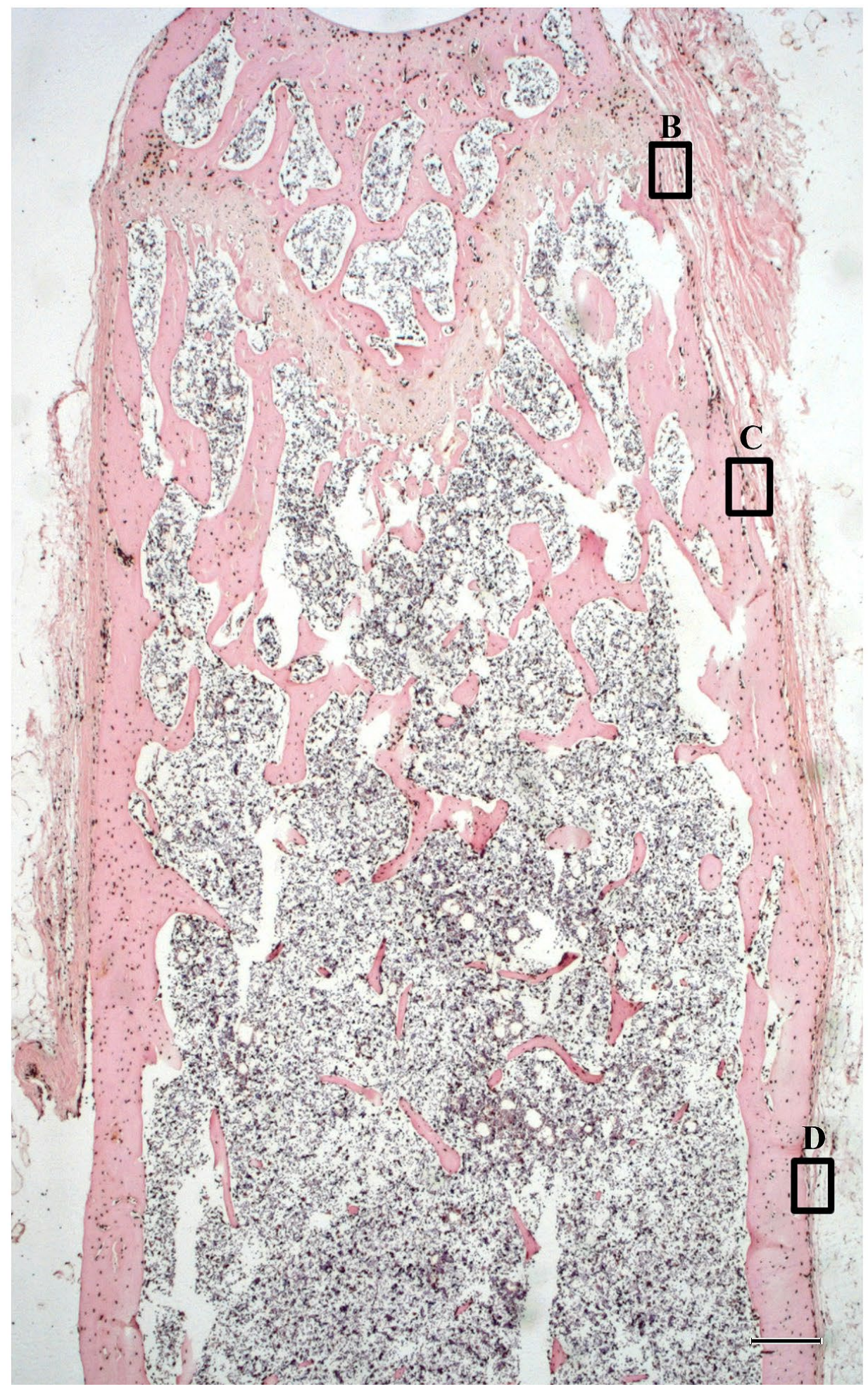

B

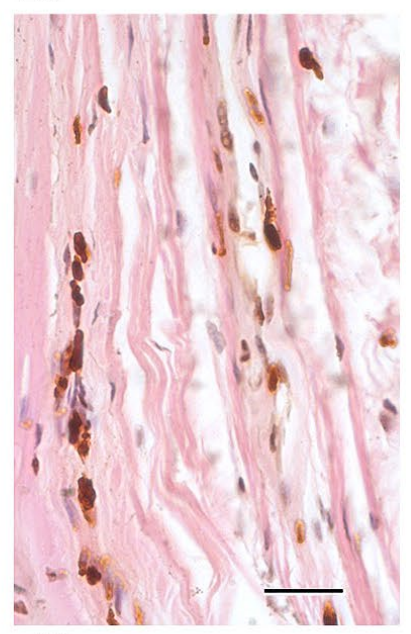

C

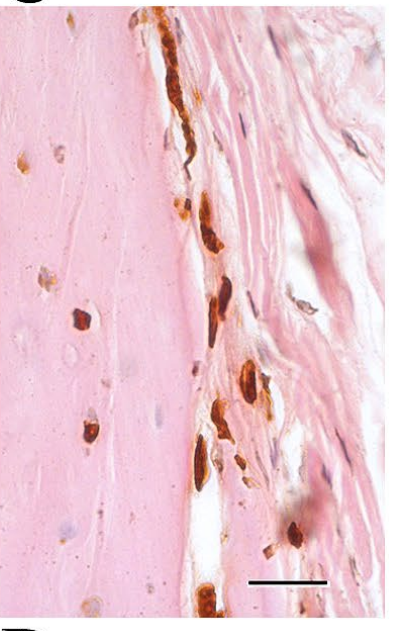

D

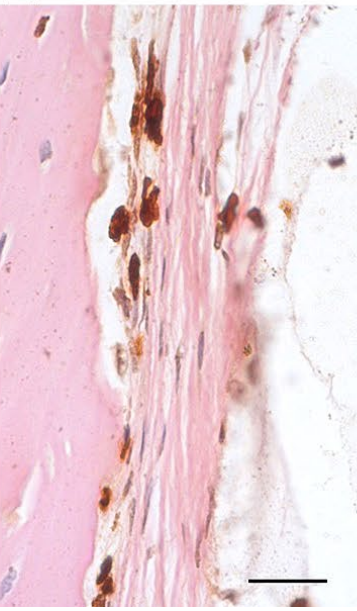

Fig. 1. Detection of nucleoside-label-retaining cells in mouse periosteum. Three-week-old C57Bl/6 mice were given the nucleoside analogue IdU for 30 days followed by a 40-day washout period. IdU-labelled cells were then detected in histological sections of the distal femur by immunohistochemistry. (A) Brightfield image acquired at 250x original magnification showing an overview of a longitudinal section through the femur immunostained for IdU. The secondary antibody was visualised using 3,3-diaminobenzidine peroxidase substrate (brown) and the section was counterstained with haematoxylin and eosin. Bar $=200 \mu \mathrm{m}$. (B-D) Higher magnification images of the boxed areas in (A) showing IdU-labelled cells (brown) in the periosteum lining the perichondrial groove of Ranvier (B), metaphysis (C), and diaphysis (D). Bars $=20 \mu \mathrm{m}$. 

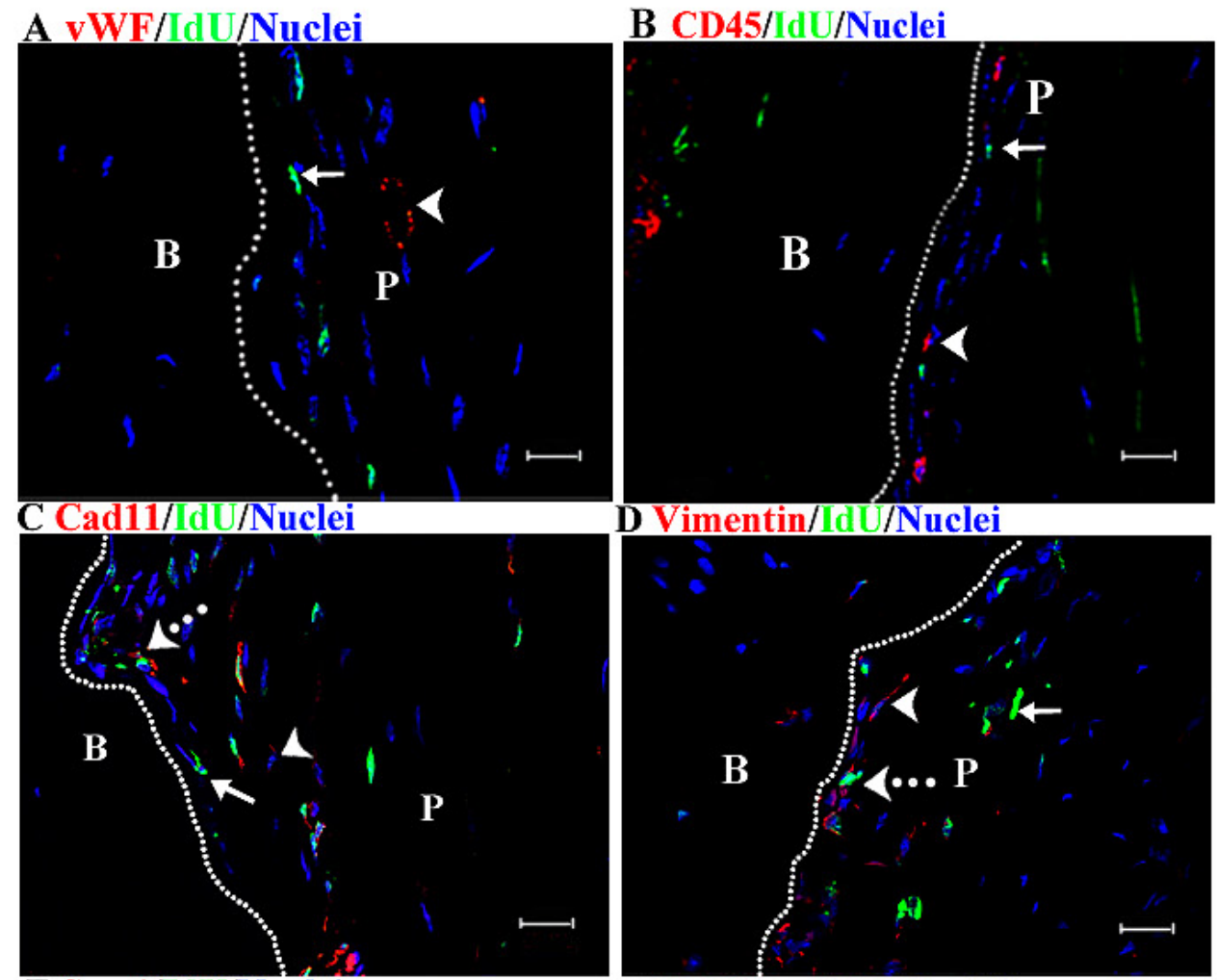

Fig. 2. Phenotypic characterisation of nucleoside-label-retaining cells in mouse periosteum Three-week-old C57Bl/6 mice were given the nucleoside analogue IdU for 30 days followed by a 40-day washout period. The phenotype of IdU-labelled cells in the periosteum lining the distal femur was determined in histological sections by double immunofluorescence staining for IdU (green) and MSC/lineage markers (red). Nuclei were counterstained with DAPI (blue). Representative images are shown for the endothelial marker vWF (A), the haematopoietic marker CD45 (B), the mesenchymal/stromal markers cadherin-11 (C) and vimentin (D), and the MSC markers Sca-1 (E), PDGFR $\alpha$ (F), p75 (G), CD44 (H), CD73 (I) and CD105 (J). Arrows indicate IdU singlepositive cells; arrowheads indicate marker singlepositive cells; dashed arrows indicate IdU and marker double-positive cells. P: periosteum; B: bone. Bars $=20 \mu \mathrm{m}$.
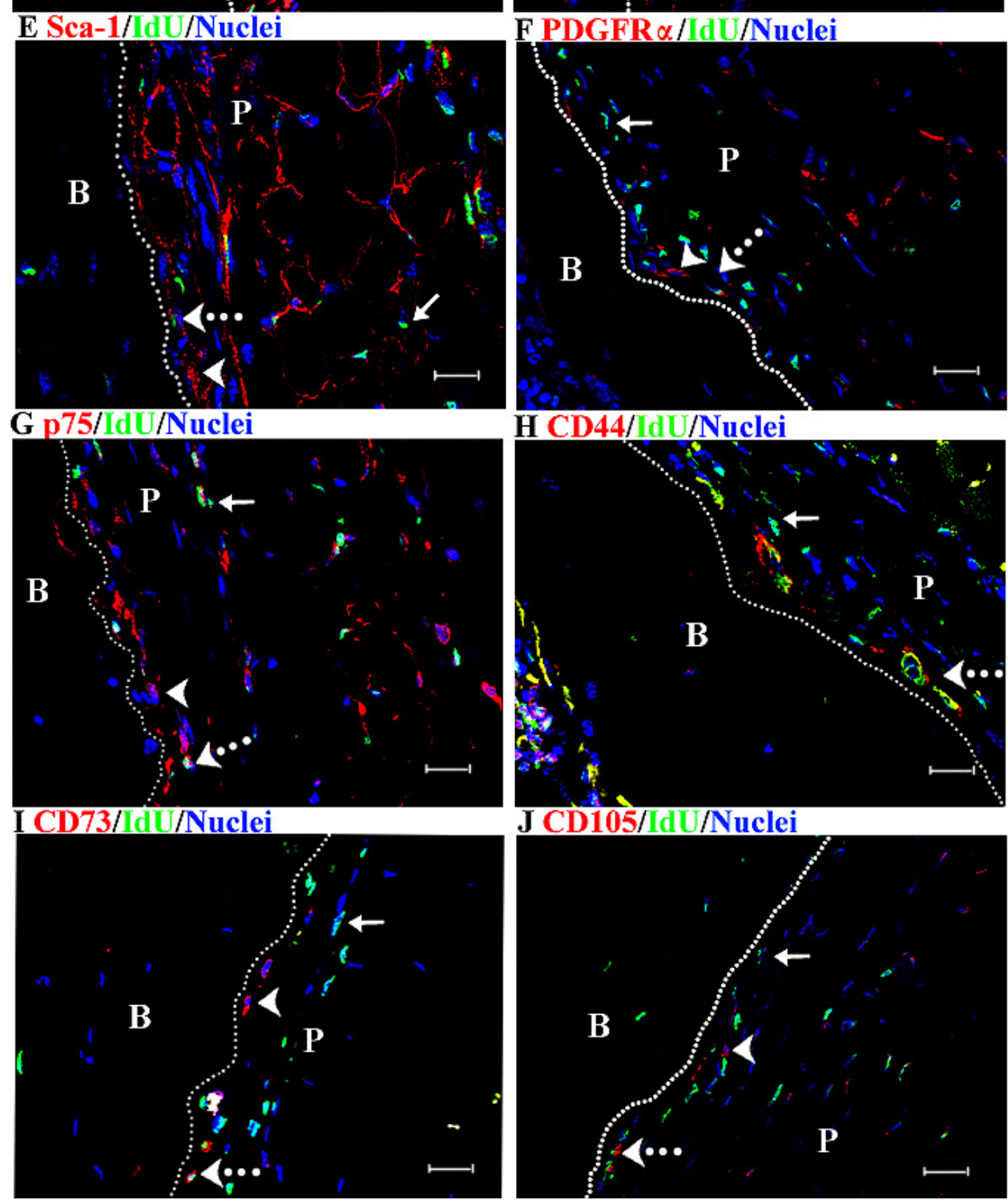


\section{A CD146/IdU/Nuclei}

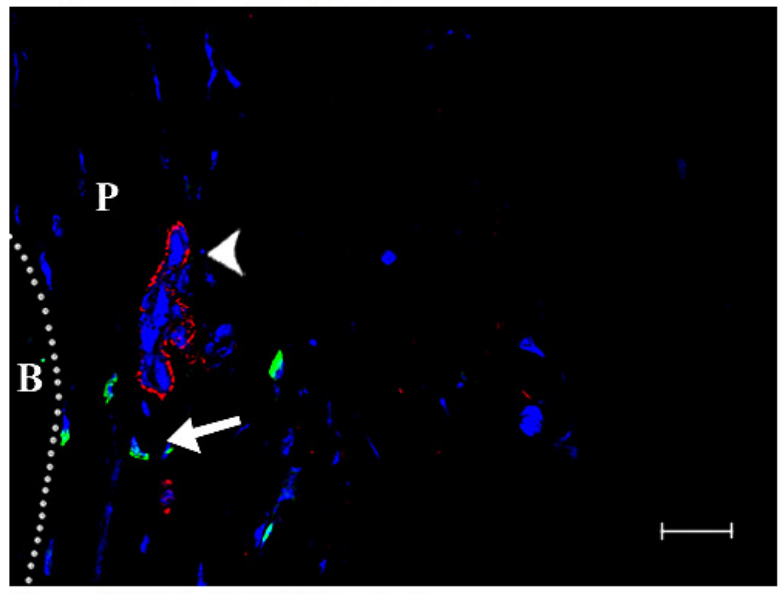

C $\boldsymbol{\alpha}$-SMA/IdU/Nuclei

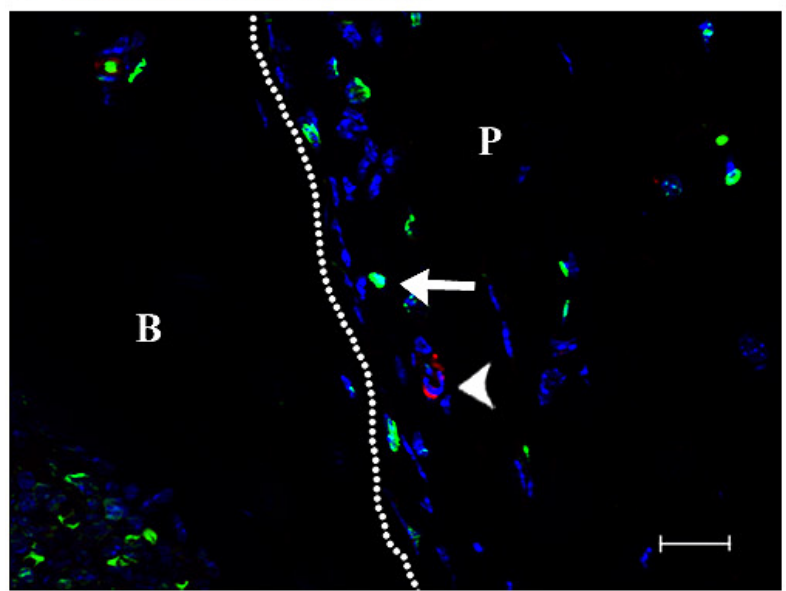

\section{E CD146/IdU/PDGFR $\alpha$}

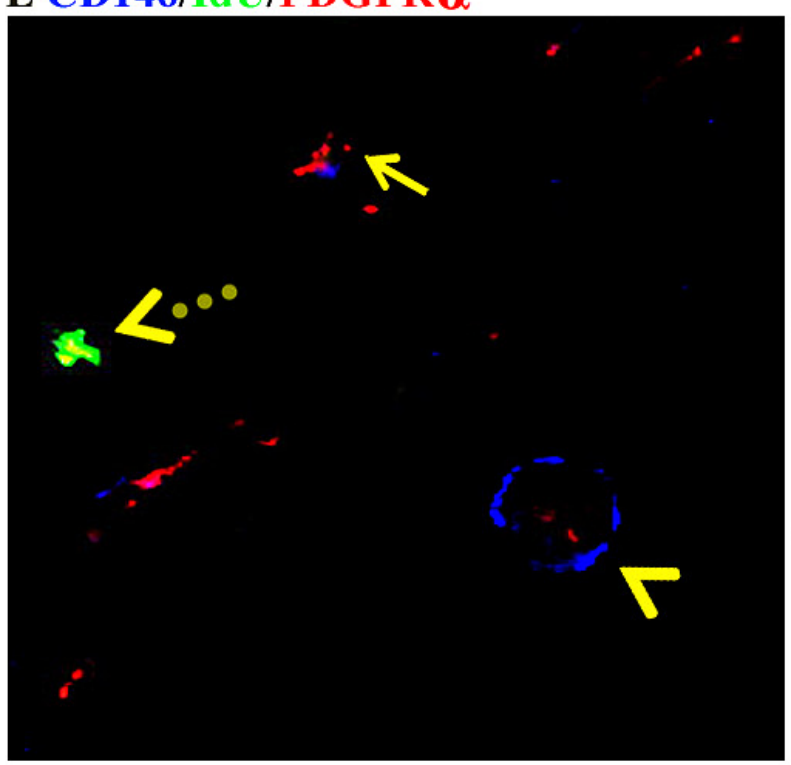

B NG2/IdU/Nuclei

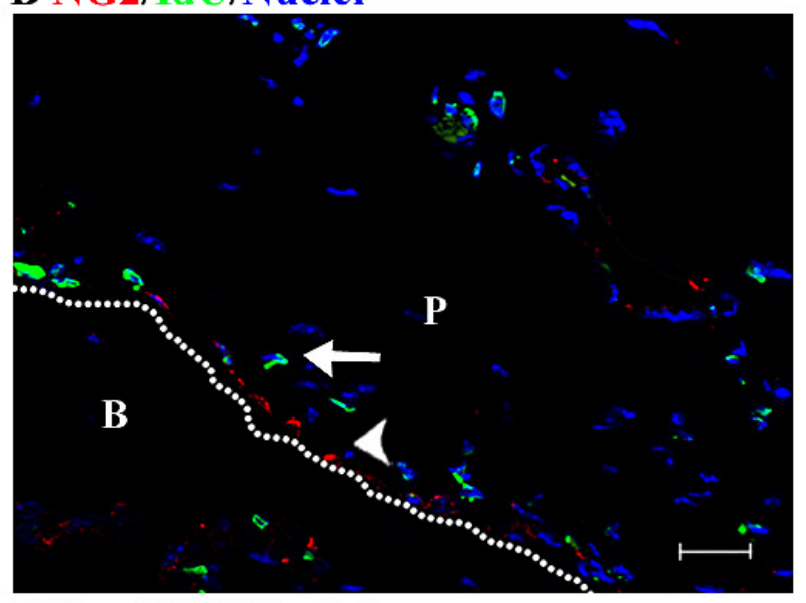

\section{D $\beta$-gal/IdU/Nuclei}

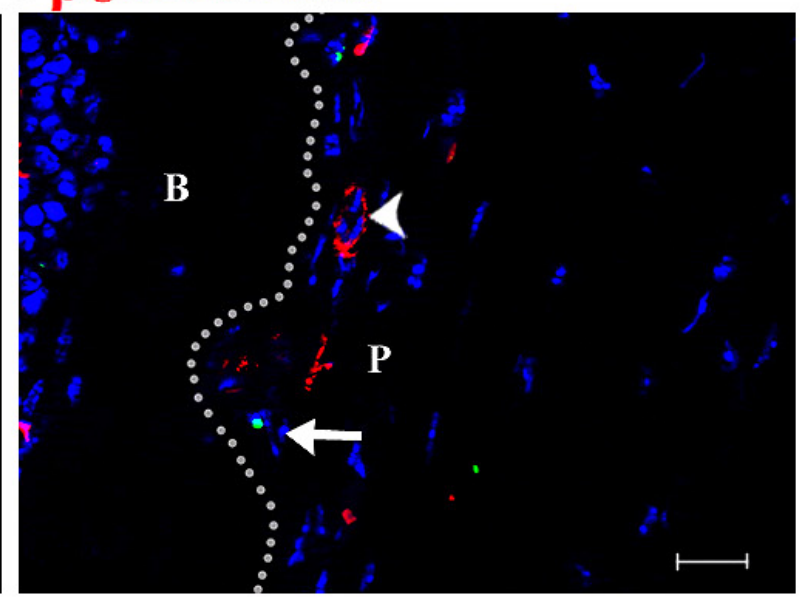

F CD146/IdU/PDGFR $\alpha /$ Nuclei (White)

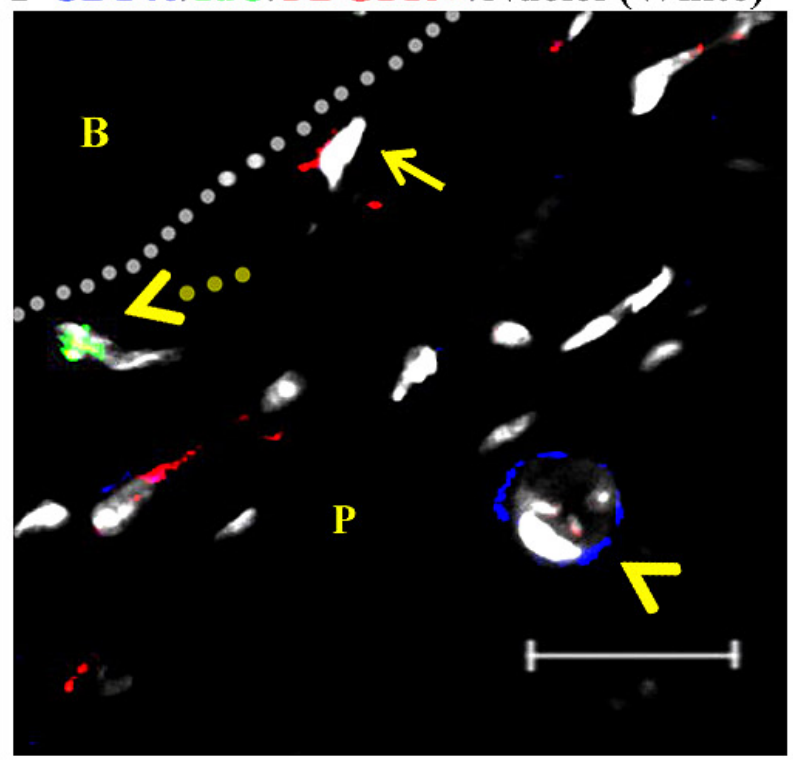

Fig. 3. Determination of nucleoside-label-retention by pericytes in mouse periosteum. Three-week-old C $57 \mathrm{Bl} / 6 \mathrm{mice}$ (A-C) and a transgenic mouse expressing B-gal in pericytes and smooth muscle cells (D) were given the nucleoside analogue IdU for 30 days followed by a 40-day washout period. Incorporation and retention of IdU by pericytes in periosteum was then determined in histological sections of the distal femur by double immunofluorescence staining for IdU (green) and the pericyte markers (red) CD146 (A), NG2 (B), and aSMA (C), or ß-gal (D). Nuclei were counterstained with DAPI (blue). Arrowhead indicating pericyte marker staining and arrow showing IdU staining. (E and F) Triple immunofluorescence staining of distal femur for the pericyte marker CD146 (blue), the MSC marker PDGFR $\alpha$ (red) and IdU (green). Nuclei were counterstained with DAPI (white). Yellow arrowhead indicates CD146 positive cell; yellow arrow shows PDGFR $\alpha$ positive cell; dotted yellow arrow points to IdU+ cell. P: periosteum; B: bone. Bars $=20 \mu \mathrm{m}$. 


\section{A RUNX2/IdU/Nuclei}

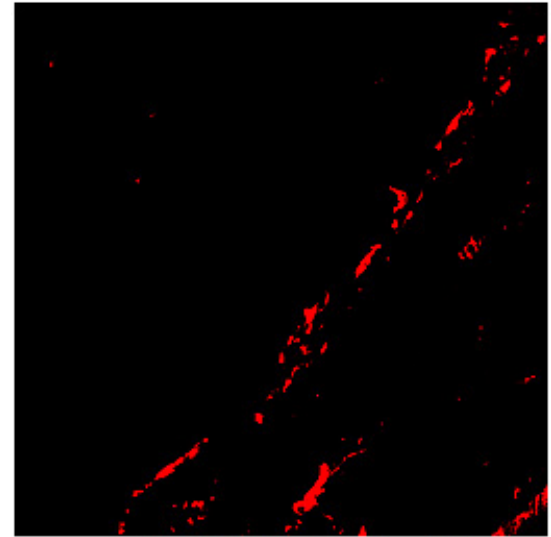

B RUNX2/p75/Nuclei

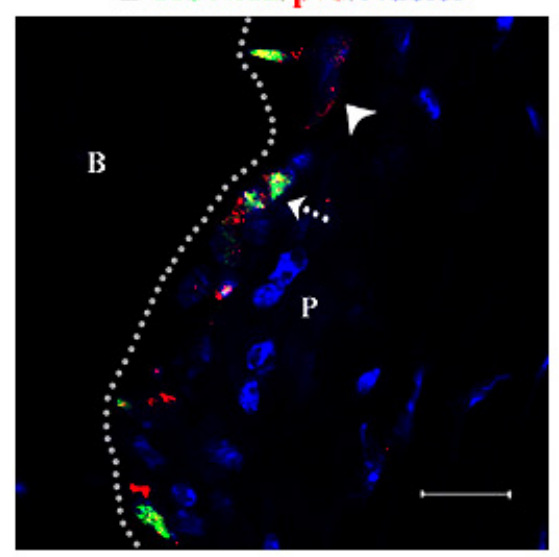

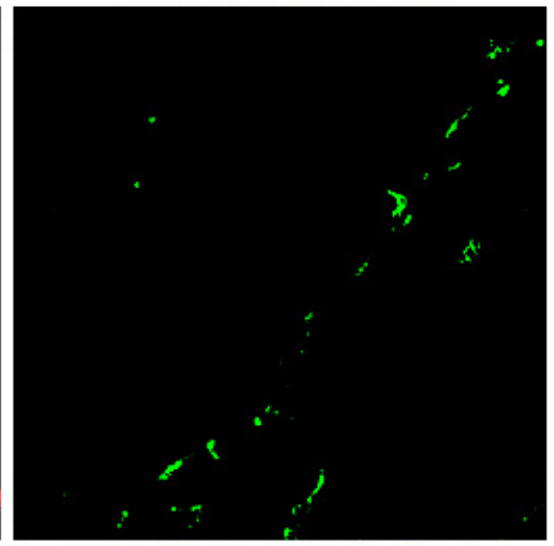

C RUNX2/PDGFR $\alpha /$ Nuclei

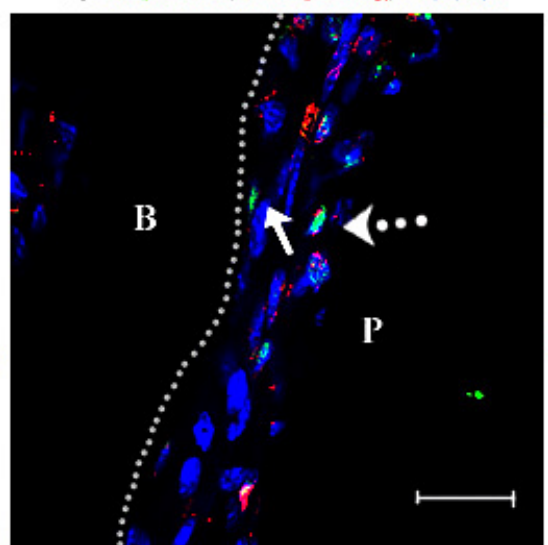

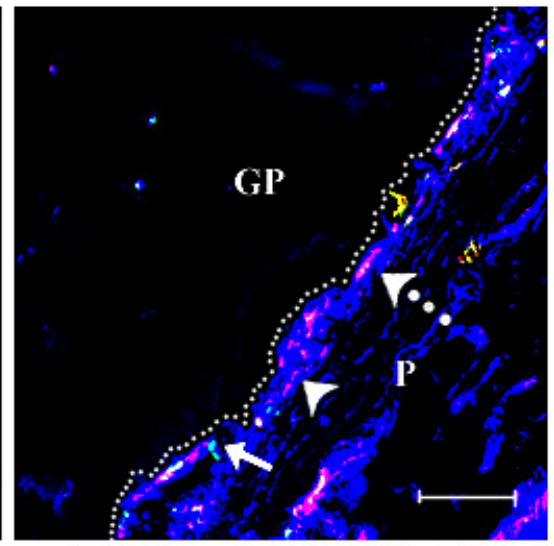

D Osteocalcin/IdU/Nuclei

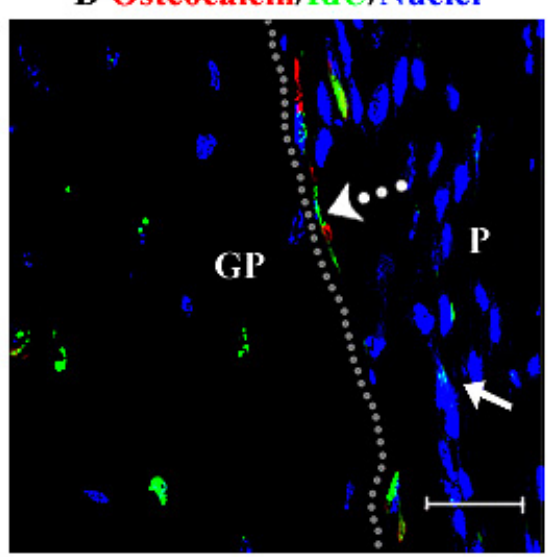

E Osteocalcin/IdU/p75
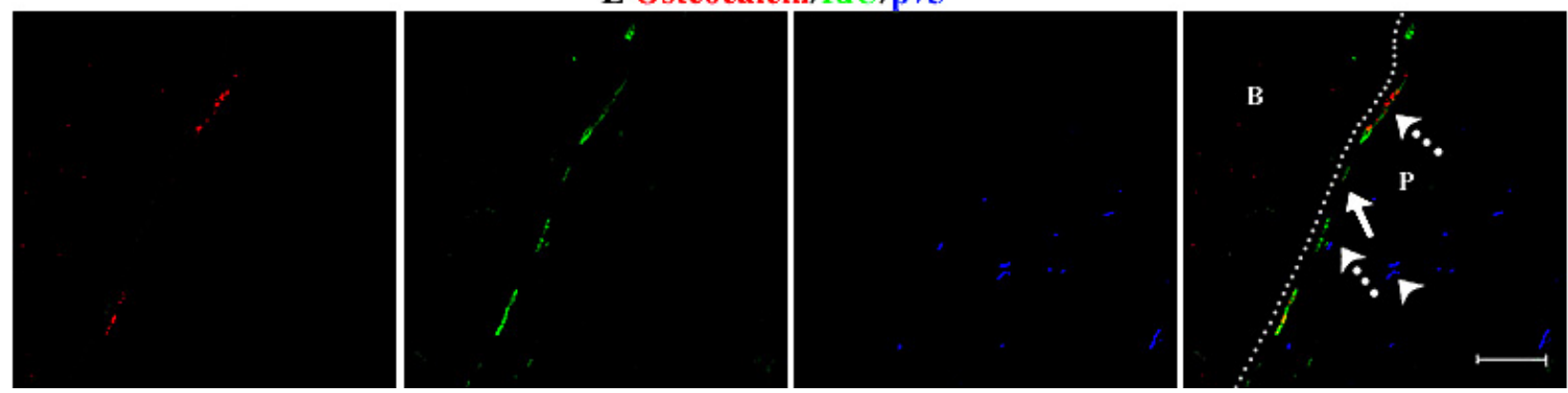

Fig. 4. Nucleoside-label-retention by osteoprogenitors and osteoblasts in mouse periosteum. Three-week-old C57B1/6 mice were given the nucleoside analogue IdU for 30 days followed by a 40-day washout period. Incorporation and retention of IdU by osteoprogenitors and osteoblasts in periosteum was then determined in histological sections of the distal femur by double immunofluorescence staining for the osteoblast markers (red) Runx2 (A) or osteocalcin (D) and IdU (green). Nuclei were counterstained with DAPI (blue). Arrows indicate IdU single-positive cells; arrowheads indicate marker single-positive cells; dashed arrows indicate IdU and marker double-positive cells. Detection of osteoprogenitor marker expression by MSC marker positive cells (B and $\mathbf{C}$ ) by double immunofluorescence staining for the early osteoblast marker Runx2 (green), the MSC marker p75 or PDGFR $\alpha$ (red). Nuclei were counterstained with DAPI (blue). Arrows indicate Runx2 single positive cells, arrowheads indicate MSC marker single positive cells and dashed arrows indicate Runx2 and MSC marker double positive cells. Triple immunofluorescence staining (E) for osteocalcin (red), IdU (green) and p75 (blue). Nuclear counterstaining is not shown for clarity. Arrows indicate IdU single-positive cells; arrowheads indicate marker single-positive cells; dashed arrows indicate either IdU-marker double-positive cells or IdU-osteocalcin double positive cells; no triple-positive cells were detected. P: periosteum; B: bone; GP: growth plate. Bars $=20 \mu \mathrm{m}$.

directly adjacent to the bone surface, as well as at some distance from the bone (Fig. 2E-G). Finally, expression of markers associated with culture-expanded MSCs (Djouad et al., 2005; Dominici et al., 2006) was investigated. CD44 (Fig. 2H) and CD73 (Fig. 2I) were found to be expressed by a subset of IdU+ cells located mostly close to the bone surface. In addition, IdU+ cells were found positive for CD105 (Fig. 2J). Similar patterns of phenotypic marker expression by IdU+ cells were found throughout the areas of periosteum analysed (i.e., perichondrial groove of Ranvier, metaphysis, and diaphysis). 


\section{Quantification of labelled cells}

For quantitative analysis of the labelled cells in mouse periosteum, we opted to use p75 as an MSC marker because other conventional markers of MSCs are known to be expressed by osteoblasts, e.g., PDGFR $\alpha$ (Finkenzeller et al., 2010), CD73 (Takedachi et al., 2011) and CD44 (Fujii et al., 2003). Cell counts of at least 500 DAPIpositive cells from at least 3 sections per mouse revealed that IdU-positive cells were $45 \pm 6 \%$, p75-positive cells were $40 \pm 14 \%$, and IdU/p 75 double-positive cells were $27 \pm 10 \%$ of the total DAPI-counterstained cell population. The proportion of cells within the IdU-positive cell pool that co-stained for the MSC marker p 75 was $58 \pm 15 \%$. The proportion of cells, within the p75-labelled cell population, positive for IdU was $68 \pm 13 \%$. These results indicate that the MSC marker p75 was not specifically expressed by IdU-positive cells, because there were cells that were positive for $\mathrm{p} 75$ but negative for IdU. In addition, p75 staining did not identify all IdU-positive cells.

\section{Nucleoside-label-retaining cells in periosteum do not express pericyte markers}

In recent years, studies have suggested that MSCs in various tissues reside in a perivascular niche and form part of the pericyte compartment (Sacchetti et al., 2007; Crisan et al., 2008). Therefore, the question whether the labelretaining cells in mouse periosteum expressed pericyte markers was investigated. As expected, cells positive for the pericyte markers CD146 and NG2 were detected in a perivascular location. However, the CD146+ and NG2+ cells had not incorporated or failed to retain the nucleoside label as they were negative for IdU (Fig. 3A,B). Similarly, alpha smooth muscle actin ( $\alpha$-SMA), expressed by human pericytes surrounding venules and arterioles (Crisan et $a l ., 2008)$ and recently proposed as a marker of osteochondroprogenitor cells in mice (Grcevic et al., 2012), did not co-localise with IdU (Fig. 3C). To further confirm these findings, IdU labelling and detection was performed in a transgenic mouse in which $\beta$-galactosidase ( $\beta$-gal) is expressed in pericytes and smooth muscle cells. This transgenic mouse line was generated by random insertional mutagenesis and the specific regulatory elements driving the expression of $\mathrm{LacZ}$ in these mice have not been identified (Tidhar et al., 2001). IdU was not detected in $\beta$-gal-expressing cells in periosteum (Fig. 3D). These data indicate that in periosteum long-term label-retaining cells, at least partly displaying an MSC-like phenotype, may be distinct from pericytes. To clarify the relationship between MSCs and pericytes in periosteum, we performed triple immunofluorescence staining for PDGFR $\alpha$, CD146 and IdU (Fig. 3E,F). CD146+ pericytes associated with the vasculature were negative for PDGFR $\alpha$. However, CD146 and PDGFR $\alpha$ double-positive cells were observed that were not associated with the vasculature. These cells did not stain for IdU, although detection of IdU was suboptimal under the conditions used.

\section{Subsets of nucleoside-label-retaining cells are osteoblasts that are distinct from MSCs}

Nucleoside-label-retention in vivo is a hallmark of stem cells, due to their slow-cycling nature. However, cells that differentiate into a mature, post-mitotic cell type may also retain the nucleoside label. Results shown in Fig. 2A,B indicated that IdU+ cells were not of the endothelial or haematopoietic lineages, respectively. To investigate whether IdU+ cells were of the osteoblastic lineage, coimmunostainings for IdU and osteoblast markers were performed. Runx2, a key transcription factor expressed early during osteogenic differentiation, was detected in subsets of IdU+ cells (Fig. 4A), and Runx2 positive cells co-stained for the MSC markers p75 or PDGFR $\alpha$, with no specific topographic distribution across the thickness of the periosteum (Fig. 4B,C). Co-staining for IdU and osteocalcin, a marker of mature osteoblasts, revealed the presence of double-positive cells which were typically in direct contact with the periosteal surface (Fig. 4D). In addition, osteocytes embedded within the cortical bone were found positive for IdU (Fig. 1). Triple immunofluorescence staining for IdU in conjunction with p75 as an MSC marker and osteocalcin as an osteoblast marker did not reveal any triple positive cells (Fig. 4E), confirming the presence of distinct populations of label-retaining cells with either an MSC or an osteoblastic phenotype.

\section{Discussion}

It is widely established that periosteum contains stem/ progenitor cells that play important roles in bone regeneration/repair. However, lack of specific markers has hindered studies identifying and characterising these cells in their native tissue. In this study, a classical long-term nucleoside-label-retaining assay was used to identify stem cells in mouse periosteum based on their slow-cycling nature combined with phenotypic marker expression. The findings reported here demonstrate the existence of labelretaining cells in periosteum with a phenotype compatible with MSCs.

Label-retaining cells were found in the distal femur of three-month-old mice throughout the areas of periosteum analysed, i.e. at the level of the growth plate, metaphysis and diaphysis. This is at odds with a recent study in rabbits in which label-retaining cells were found to be concentrated in the perichondrial groove of Ranvier (Karlsson et al., 2009). This discrepancy may reflect species or age differences, as in the Karlsson study BrdU was administered to sexually mature rabbits from 3 months of age, while in the present study IdU was administered to mice from 3 weeks of age, during active bone growth. At time of analysis the mice were around 13 weeks of age, but periosteal bone apposition continues at this age through direct membranous ossification to widen the bone. The presence of a large proportion of label-retaining cells throughout periosteum may thus reflect a high rate of periosteal bone formation in these mice. Our quantification data also suggests the heterogeneity of the slow-cycling cells in mouse periosteum as not all IdU-positive cells were positive for the MSC marker p75. Here we used p75 as the only MSC marker because other MSC markers such as PDGFR $\alpha$ (Finkenzeller et al., 2010), CD73 (Takedachi et al., 2011) and CD44 (Fujii et al., 2003) are reported to be also expressed by osteoblasts. 
In our study, we observed a similar distribution of slowcycling cells throughout the periosteum, irrespective of their anatomical location. One reason could be the young age of the mice during the nucleoside labelling. It may be that there is an active contribution of MSCs from all over the periosteum to growth.

Irrespective of the anatomical area, IdU-retaining cells were frequently located close to the bone surface, presumably within the cambium layer. This is consistent with the known presence of osteo-chondroprogenitor cells in the cambium layer of the periosteum (Tonna and Cronkite, 1962; Ito et al., 2001). Extensive phenotypic marker analyses demonstrated the existence of IdUretaining cells in periosteum with a phenotype compatible with MSCs. Similar to our recent study in synovium (Kurth et al., 2011), none of the MSC markers tested labelled all the IdU-retaining cells and all markers tested also labelled IdU-negative cells.

Of note, IdU+ cells in periosteum were found positive for CD105, an established marker of human MSCs in culture (Dominici et al., 2006). This finding is in contrast to synovium, where we previously failed to detect IdUretaining cells expressing CD105 (Kurth et al., 2011). This could reflect an interesting phenotypic or even functional difference between MSCs from these two tissues, although we cannot exclude the possibility that CD105 labelled (an) other label-retaining cell type(s) within periosteum. In this regard, CD105 is also expressed by endothelial cells but the undetectable co-staining between IdU and vWF would make an endothelial nature of IdU+CD105+ cells unlikely.

Nucleoside-label-retaining assays can, in addition to slow-cycling stem cells, also label mature, post-mitotic cell types. Indeed, epiphyseal chondrocytes were found labelled with IdU. Labelling of chondrocytes was most notable towards the outer edges of the growth plate. These findings are consistent with the Karlsson study (Karlsson et al., 2009) and are in keeping with the view that cells in the perichondrial groove of Ranvier may act as stem/ progenitor cells for epiphyseal chondrocytes during bone growth (Shapiro et al., 1977), a view that is supported by the observation that removal of the perichondrial groove of Ranvier results in impaired longitudinal bone growth (Rodriguez et al., 1985). In addition, subsets of osteoblasts in periosteum and osteocytes in cortical bone were found positive for the nucleoside label. However, label-retaining osteoblasts were negative for the MSC marker p75, confirming that at least part of the MSC-like label-retaining cells that were identified were distinct from osteoblasts. In the adult skeleton, MSCs in periosteum may continuously replenish a pool of short-lived osteoblasts contributing to bone remodelling and repair throughout life, as was recently demonstrated in bone marrow (Park et al., 2012). In addition to providing a reservoir of precursors for mature skeletal cells, MSCs may also play essential roles in maintaining the periosteal stroma/vasculature, similar to MSCs found in bone marrow (Sacchetti et al., 2007; Mendez-Ferrer et al., 2010).

Increasing evidence suggests that MSCs reside in a perivascular niche in various tissues and organs and form part of the pericyte compartment (Shi and Gronthos, 2003; Dellavalle et al., 2007; Sacchetti et al., 2007; Crisan et al.,
2008; Feng et al., 2011; Grcevic et al., 2012). Therefore, the question whether the label-retaining cells in periosteum displayed a pericyte phenotype was investigated. Under the experimental conditions used, very little or no colocalisation was detected between IdU and the pericyte markers CD146, NG2 and $\alpha$-sma, nor with $\beta$-gal in a transgenic mouse in which this reporter gene is expressed by pericytes and smooth muscle cells. We previously obtained similar findings in mouse synovium, where we further demonstrated that pericytes, in contrast to labelretaining MSCs, did not proliferate in response to joint surface injury, indicating these cells did not behave like MSCs, at least in this model (Kurth et al., 2011).

The findings of the current study seem at odds with a recent study by Grcevic et al. (2012) who demonstrated through lineage tracing that progeny of $\alpha$-sma + cells give rise to trabecular osteoblasts during normal bone formation and to both chondrocytes and osteoblasts during fracture repair (Grcevic et al., 2012). Although it seems intuitive that in the study by Grcevic et al. progeny of $\alpha$-sma+ cells in periosteum contributed to the formation and subsequent remodelling of the fracture callus, their periosteal origins remain to be proven. It is possible that labelled progeny of $\alpha$-sma + cells in the fracture callus were derived from bone marrow or other tissues. An alternative explanation may be that different populations of MSCs exist in periosteum, some of which are pericytes or are derived from pericytes, but that this population of pericyte-like MSCs had not efficiently incorporated and/or retained the nucleoside label in our study due to differences in cycling rates.

It appears from our data that there may be tissue heterogeneity in the contribution of pericytes to the MSC pool. This is supported by a study showing that only a minority of odontoblasts originate from pericytes, both during tooth development and in response to injury (Feng et al., 2011). The paradigm that has emerged in recent years of the pericyte origin of MSCs may thus not be universally applicable to all tissues or experimental models. The relative contributions from the pericyte compartment to the MSC pool may reflect distinct ontogeny paths of MSCs in different tissues, and/or tissue-specific functions of MSC-like cells, such as the support of the perivascular haematopoietic stem cell (HSC) niche and sinusoidal network in bone marrow (Sacchetti et al., 2007; MéndezFerrer et al., 2010,).

The topography of periosteal MSCs is largely unknown, but earlier reports based on morphological analysis of label-retaining cells (Tonna and Cronkite et al., 1962) and analysis of cartilage formation in periosteal explant cultures (Ito et al., 2001) suggested that cells in periosteum closest to the bone surface are more differentiated, committed progenitors, while undifferentiated stem cells reside in the area of the cambium layer that is farthest from the bone. This is an attractive model, as it would suggest a flow of stem/progenitor cells from the outer cambium inward, with progressively increased levels of lineage commitment/differentiation as the cells migrate closer to the bone, until they give rise to mature osteoblasts residing on the periosteal surface. However, the phenotypic marker analyses carried out in the current study revealed the presence of label-retaining cells expressing MSC markers 
in close proximity to bone. These findings appear not in keeping with the earlier proposed model, although one possible explanation could be that the nucleoside labelling scheme used in the present study, in addition to labelling MSCs, may have labelled committed progenitors that still express some MSC markers. In support of this, we detected Runx2 positive osteoprogenitors, which were also positive for the MSC markers p75 or PDGFR $\alpha$.

Adult stem cells are traditionally considered to be slow-cycling, label-retaining cells that give rise to transit-amplifying progenitor cells which in turn give rise to daughter cells that differentiate into mature cell types. However, in the haematopoietic system, significant heterogeneity exists in the cycling rates of otherwise phenotypically identical HSCs. Recent studies demonstrated that under homeostatic conditions, around one-fifth of HSCs proliferate at an extremely low rate, estimated at $\leq 0.8-1.8 \%$ per day (Foudi et al., 2009), or around once every 145 days (Wilson et al., 2008). Dormant and active HSCs are thought to reside in distinct topographical niches in the bone marrow, namely the endosteal and perivascular niche, respectively (Celso and Scadden, 2011). Similarly, increasing evidence indicates that dormant and active stem cells co-exist in other adult tissues, including the intestinal crypts, the bulge of the hair follicle, and possibly the subventricular zone of the brain, in adjacent but distinct niches (Greco and Guo, 2010; Li and Clevers, 2010). It is postulated that quiescent stem cells with very low cycling rates represent a population of the most primitive, undifferentiated stem cells which ensure lifelong maintenance of the stem cell compartment, while faster cycling stem cells are responsible for supplying the continuous pool of progenitor cells required for normal tissue turnover.

It is tempting to speculate that, similar to other stem cell types, MSCs may be heterogeneous in their cycling rates under homeostatic conditions. If the most dormant, undifferentiated MSCs cycle at similar rates as the most dormant HSC population currently identified (i.e., around once every 4-5 months (Wilson et al., 2008; Foudi et al., 2009), the label-retaining assay employed in the current study would have failed to label the majority of these cells.

Furthermore, it has been postulated that stem cells, during asymmetric cell division, retain the original set of DNA strands while the newly synthesised strands are passed on to differentiating daughter cells. This is known as the immortal strand hypothesis (Cairns, 1975). Such non-random segregation of DNA strands would alter the interpretation of the label-retaining assays. However, although some evidence in support of the immortal strand hypothesis exists in other stem cell types, it remains unclear whether this applies to all stem cells in all tissues (Yadlapalli et al., 2008). In addition, it is currently not known under what conditions MSCs divide asymmetrically. Therefore, whether we have identified the most primitive stem cells remains to be clarified.

In conclusion, we have identified and phenotypically characterised label-retaining cells in mouse periosteum and have demonstrated the existence of label-retaining cells with a phenotype compatible with MSCs. Further studies will be required to demonstrate whether the model described above, i.e. that the most primitive MSCs are located furthest from bone, may hold true. Such studies will shed light on the spatial and temporal relationships between MSCs, progenitors, and mature cell types, in periosteum and indeed other tissues. A full understanding of the biology of multipotent stem/progenitor cells in periosteum is critical for the development of improved protocols and discovery of novel pharmacological targets to maintain bone homeostasis throughout life and stimulate bone regeneration and repair.

\section{Acknowledgements}

We are grateful to members of the Regenerative Medicine Group and Musculosketelal Research Programme, and in particular Dr Andrea Augello, for help and advice. This work was supported by the Islamic Development Bank and Arthritis Research UK (grants 19271 and 19429). The funding sources had no involvement in the study design, the collection, analysis and interpretation of data, the writing of the report, or the decision to submit the article for publication.

\section{References}

Celso CL, Scadden DT (2011) The haematopoietic stem cell niche at a glance. J Cell Sci 124: 3529-3535.

Cotsarelis G, Sun TT, Lavker RM (1990) Labelretaining cells reside in the bulge area of pilosebaceous unit: implications for follicular stem cells, hair cycle, and skin carcinogenesis. Cell 61: 1329-1337.

Crisan M, Yap S, Casteilla L, Chen C-, Corselli M, Park TS, Andriolo G, Sun B, Zheng B, Zhang L, Norotte C, Teng P-, Traas J, Schugar R, Deasy BM, Badylak S, Buhring HJ, Giacobino JP, Lazzari L, Huard J, Péault B (2008) A perivascular origin for mesenchymal stem cells in multiple human organs. Cell Stem Cell 3: 301-313.

De Bari C, Dell'Accio F, Luyten FP (2001) Human periosteum-derived cells maintain phenotypic stability and chondrogenic potential throughout expansion regardless of donor age. Arthritis Rheum 44: 85-95.

De Bari C, Dell'Accio F, Vanlauwe J, Eyckmans J, Khan IM, Archer CW, Jones EA, McGonagle D, Mitsiadis TA, Pitzalis C, Luyten FP (2006) Mesenchymal multipotency of adult human periosteal cells demonstrated by single-cell lineage analysis. Arthritis Rheum 54: 1209-1221.

Dellavalle A, Sampaolesi M, Tonlorenzi R, Tagliafico E, Sacchetti B, Perani L, Innocenzi A, Galvez BG, Messina G, Morosetti R, Li S, Belicchi M, Peretti G, Chamberlain JS, Wright WE, Torrente Y, Ferrari S, Bianco P, Cossu G (2007) Pericytes of human skeletal muscle are myogenic precursors distinct from satellite cells. Nat Cell Biol 9: 255-267.

Díaz-Prado S, Muiños-López E, Hermida-Gómez T, Rendal-Vázquez ME, Fuentes-Boquet I, de Toro FJ, Blanco FJ (2010) Multilineage differentiation potential of cells isolated from the human amniotic membrane. J Cell Biochem 111: 846-857. 
Djouad F, Bony C, Haupl T, Uze G, Lahlou N, Louis-Plence P, Apparailly F, Canovas F, Reme T, Sany J, Jorgensen C, Noel D (2005) Transcriptional profiles discriminate bone marrow-derived and synovium-derived mesenchymal stem cells. Arthritis Res Ther 7: R13041315.

Dominici M, Le Blanc K, Mueller I, Slaper-Cortenbach I, Marini F, Krause D, Deans R, Keating A, Prockop D, Horwitz E (2006) Minimal criteria for defining multipotent mesenchymal stromal cells. The International Society for Cellular Therapy position statement. Cytotherapy 8: 315317.

Dou C, Yan Y, Dong S (2012) Role of cadherin-11 in synovial joint formation and rheumatoid arthritis pathology. Mod Rheumatol 23: 1037-1044.

Feng J, Mantesso A, De Bari C, Nishiyama A, Sharp PT (2011) Dual origin of mesenchymal stem cells contributing to organ growth and repair. Proc Natl Acad Sci USA 108: 6503-6508.

Finkenzeller G, Mehlhorn AT, Schmal H, Stark GB (2010) Post-transcriptional regulation of osteoblastic platelet-derived growth factor receptor-alpha expression by co-cultured primary endothelial cells. Cells Tissues Organs 192: 28-38.

Foudi A, Hochedlinger K, Van Buren D, Schindler JW, Jaenisch R, Carey V, Hock H (2009) Analysis of histone 2B-GFP retention reveals slowly cycling hematopoietic stem cells. Nat Biotechnol 27: 84-90.

Fujii Y, Fujii K, Nakano K, Tanaka Y(2003) Crosslinking of CD44 on human osteoblastic cells upregulates ICAM-1 and VCAM-1. FEBS Lett 539:45-50.

Gerstenfeld LC, Cullinane DM, Barnes GL, Graves DT, Einhorn TA (2003) Fracture healing as a post-natal developmental process: molecular, spatial, and temporal aspects of its regulation. J Cell Biochem 88: 873-884.

Grcevic D, Pejda S, Matthews BG, Repic D, Wang L, Li H, Kronenberg MS, Jiang X, Maye P, Adams DJ, Rowe DW, Aguila HL, Kalajzic I (2012) In vivo fate mapping identifies mesenchymal progenitor cells. Stem Cells 30: 187-196.

Greco V, Guo S (2010) Compartmentalized organization: A common and required feature of stem cell niches? Development 137: 1586-1594.

Grellier M, Bordenave L, Amédée J (2009) Cell-tocell communication between osteogenic and endothelial lineages: implications for tissue engineering. Trends Biotechnol. 27: 562-571.

Guler G, Balci S, Costinean S, Ussakli CH, Irkkan C, Suren D, Sari E, Altundag K, Ozisik Y, Jones S, Bacher J, Shapiro CL, Huebner K (2012) Stem cell-related markers in primary breast cancers and associated metastatic lesions. Mod Pathol 25: 949-955.

Ito Y, Fitzsimmons JS, Sanyal A, Mello MA, Mukherjee N, O'Driscoll SW (2001) Localization of chondrocyte precursors in periosteum. Osteoarthritis Cartilage 9: 215223.

Jilka RL, O’Brien CA, Ali AA, Roberson PK, Weinstein RS, Manolagas SC (2009) Intermittent PTH stimulates periosteal bone formation by actions on post-mitotic preosteoblasts. Bone 44:275-286.
Jones EA, Kinsey SE, English A, Jones RA, Straszynski L, Meredith DM, Markham AF, Jack A, Emery P, McGonagle D (2002) Isolation and characterization of bone marrow multipotential mesenchymal progenitor cells. Arthritis Rheum 46: 3349-3360.

Karlsson C, Thornemo M, Henriksson HB, Lindahl A (2009) Identification of a stem cell niche in the zone of Ranvier within the knee joint. J Anat 215: 355-363.

Kaur H, Phillips-Mason PJ, Burden-Gulley SM, Kerstetter-Fogle AE, Basilion JP, Sloan AE, Brady-Kalnay SM (2012) Cadherin-11, a marker of the mesenchymal phenotype, regulates glioblastoma cell migration and survival in vivo. Mol Cancer Res 10: 293-304.

Kurth TB, Dell'accio F, Crouch V, Augello A, Sharpe PT, De Bari C (2011) Functional mesenchymal stem cell niches in adult mouse knee joint synovium in vivo. Arthritis Rheum 63: 1289-1300.

Li N, Clevers H (2010) Coexistence of quiescent and active adult stem cells in mammals. Science 327: 542-545.

Méndez-Ferrer S, Michurina TV, Ferraro F, Mazloom AR, MacArthur BD, Lira SA, Scadden DT, Mag'Ayan A, Enikolopov GN, Frenette PS (2010) Mesenchymal and haematopoietic stem cells form a unique bone marrow niche. Nature 466: 829-834.

Morikawa S, Mabuchi Y, Kubota Y, Nagai Y, Niibe K, Hiratsu E, Suzuki S, Miyauchi-Hara C, Nagoshi N, Sunabori T, Shimmura S, Miyawaki A, Nakagawa T, Suda T, Okano H, Matsuzaki Y (2009) Prospective identification, isolation, and systemic transplantation of multipotent mesenchymal stem cells in murine bone marrow. J Exp Med 206: 2483-2496.

Nakahara H, Bruder SP, Goldberg VM, Caplan AI (1990) In vivo osteochondrogenic potential of cultured cells derived from the periosteum. Clin Orthop Relat Res 259: 223-232.

Nakahara H, Dennis JE, Bruder SP, Haynesworth SE, Lennon DP, Caplan AI (1991) In vitro differentiation of bone and hypertrophic cartilage from periosteal-derived cells. Exp Cell Res 195: 492-503.

Ozaki A, Tsunoda M, Kinoshita S, Saura R (2000) Role of fracture hematoma and periosteum during fracture healing in rats: interaction of fracture hematoma and the periosteum in the initial step of the healing process. J Orthop Sci 5: 64-70.

Ollier L (1867) Traite experimental et clinque de la régénération des os et de la production artificielle du tissu osseux (Experimental and clinical treatise on bone regeneration and the artificial production of bone tissue). Masson \& Fils, Paris, vol. 1.

Park D, Spencer JA, Koh BI, Kobayashi T, Fujisaki J, Clemens TL, Lin CP, Kronenberg HM, Scadden DT (2012) Endogenous bone marrow MSCs are dynamic, fate-restricted participants in bone maintenance and regeneration. Cell Stem Cell. 10: 259-272.

Peister A, Mellad JA, Larson BL, Hall BM, Gibson LF, Prockop DJ (2004) Adult stem cells from bone marrow (MSCs) isolated from different strains of inbred mice vary in surface epitopes, rates of proliferation, and differentiation potential. Blood 103: 1662-1668. 
Potten CS, Owen G, Booth D (2002) Intestinal stem cells protect their genome by selective segregation of template DNA strands. J Cell Sci 115: 2381-2388.

Qian H, Le Blanc K, Sigvardsson M (2012) Primary mesenchymal stem and progenitor cells from bone marrow lack expression of CD44 protein. J Biol Chem 287: 25795 25807.

Rodriguez JI, Delgado E, Paniagua R (1985) Changes in young rat radius following excision of the perichondrial ring. Calcif Tissue Int 37: 677-683.

Sacchetti B, Funari A, Michienzi S, Di Cesare S, Piersanti S, Saggio I, Tagliafico E, Ferrari S, Robey PG, Riminucci M, Bianco P (2007) Self-renewing osteoprogenitors in bone marrow sinusoids can organize a hematopoietic microenvironment. Cell 131: 324-336.

Shapiro F, Holtrop ME, Glimcher MJ (1977) Organization and cellular biology of the perichondrial ossification groove of Ranvier. A morphological study in rabbits. Journal of Bone and Joint Surgery - Series A 59: 703-723.

Shi S, Gronthos S (2003) Perivascular niche of postnatal mesenchymal stem cells in human bone marrow and dental pulp. J Bone Miner Res 18: 696-704.

Takedachi M, Oohara H, Smith BJ, Iyama M, Kobashi M, Maeda K, Long CL, Humphrey MB, Stoecker BJ, Toyosawa S, Thompson LF, Murakami S (2012) CD73generated adenosine promotes osteoblast differentiation. J Cell Physiol 227: 2622-2631.

Tidhar A, Reichenstein M, Cohen D, Faerman A, Copeland NG, Gilbert DJ, Jenkins NA, Shani M (2001) A novel transgenic marker for migrating limb muscle precursors and for vascular smooth muscle cells. Dev Dyn 220: $60-73$.

Tonna EA (1960) Periosteal osteoclasts, skeletal development and ageing. Nature 185: 405-407.

Tonna EA, Cronkite EP (1962) An autoradiographic study of periosteal cell proliferation with tritiated thymidine. Lab Invest 11: 455-462.

Tonna EA, Cronkite EP (1968) Skeletal cell labeling following continuous infusion with tritiated thymidine. Lab Invest 19: 510-515.

Tormin A, Li O, Brune JC, Walsh S, Schutz B, Ehinger M, Ditzel N, Kassem M, Scheding S (2011) CD146 expression on primary nonhematopoietic bone marrow stem cells is correlated with in situ localization. Blood 117: 5067-5077.

Tumbar T, Guasch G, Greco V, Blanpain C, Lowry WE, Rendl M, Fuchs E (2004) Defining the epithelial stem cell niche in skin. Science 303: 359-363.

Van de Rijn M, Heimfeld S, Spangrude GJ, Weissman IL (1989) Mouse hematopoietic stem-cell antigen Sca-1 is a member of the Ly-6 antigen family. Proc Natl Acad Sci USA 86: 4634-4638.

Wilson A, Laurenti E, Oser G, van der Wath RC, Blanco-Bose W, Jaworski M, Offner S, Dunant CF, Eshkind L, Bockamp E, Lió P, MacDonald HR, Trumpp A (2008)
Hematopoietic stem cells reversibly switch from dormancy to self-renewal during homeostasis and repair. Cell 135: 1118-1129.

Yadlapalli S, Yamashita YM (2013)DNA asymmetry in stem cells - immortal or mortal? J Cell Sci. 126: 40694076.

\section{Discussion with Reviewers}

I. Khan: Therapeutic applications utilising periosteal MSCs are likely to require in vitro expansion; can the phenotype, and thus their efficacy, be retained in vitro? And in this regard, to what extent are the in vivo non-cellular components of the periosteum integral to the phenotype of the periosteal MSCs and can we draw lessons for in vitro expansion?

Authors: We agree with the reviewer that the therapeutic use of periosteal MSCs is likely to require in vitro expansion, while ensuring that phenotype and potency of the MSCs are maintained. Our study identifying labelretaining MSCs in the periosteum is a first step towards a better understanding of the native MSCs at their niche sites. The investigation of niche components with emphasis onto the extracellular matrix could elucidate the type of environment that would be needed for three-dimensional in vitro expansion of periosteal MSCs to ensure they maintain their original phenotype and function. This would enhance consistency of bioprocessing of periosteal MSCs for clinical use.

I. Khan: It is interesting that the periosteal MSC population may be distinct from the pericytes. Could there be a potential mechanistic relationship between periosteal MSCs and the pericytes in bone formation (or fracture healing) with the pericytes supporting osteogenesis via vascularisation? If so, would therapeutic applications benefit from dual-cell implantation?

Authors: A potential mechanistic relationship between periosteal MSCs and the pericytes in bone formation or repair with the pericytes supporting osteogenesis via vascularisation is fascinating and requires investigation. Vascularisation is an important feature of bone formation/ repair, and there is evidence that endothelial cells can enhance bone formation (Grellier et al., 2009, additional reference). Dual implantation of pericytes and MSCs could thus be beneficial since pericytes are known to play an important role in vascularisation.

\section{Additional Reference}

Grellier M, Bordenave L, Amédée J (2009) Cell-tocell communication between osteogenic and endothelial lineages: implications for tissue engineering. Trends Biotechnol. 27: 562-571. 